\title{
XXII. On the problems of temperature radiation of gases. (Paper C.)
}

\section{Megh Nad Saha D.Sc.}

To cite this article: Megh Nad Saha D.Sc. (1921) XXII. On the problems of temperature radiation of gases. (Paper C.), Philosophical Magazine Series 6, 41:242, 267-278, DOI: 10.1080/14786442108636217

To link to this article: http://dx.doi.org/10.1080/14786442108636217

曲 Published online: 08 Apr 2009.

Submit your article to this journal $\sqsubset \pi$

Џ Article views: 5

Q View related articles $\square$

Citing articles: 5 View citing articles 5 
XXII. On the Problems of Temperature Radiution of Gases. (Paper C.) By MeGH NAD $\mathrm{S}_{\triangle \mathrm{HA}}$, D.Sc., Lecturer in Physics and Applied Mathematics, University College of Science, Calcutta* .

$$
\S 1 .
$$

THE object of the present paper is to discuss and examine the present-day position of the question of temperature radiation of gases. The problem before us is, whether by simply heating a quantity of gas confined within a closed vessel (say, a silica or a graphite tube), it is possible to make the gas emit its characteristic line-radiation. The experimental results on this subject are somewhat conflicting, and for different elements are widely divergent. While Pringsheim and others $\dagger$ found that permanent gases like $\mathrm{H}_{2}, \mathrm{He}, \mathrm{Ne}, \mathrm{A}, \mathrm{N}_{2}, \mathrm{O}_{2}$, etc., remain non-luminous even at the highest temperatures which can be commanded in the laboratory, it is known that vapours of many elements easily become luminous at moderate temperatures. Such, for example, are the vapours of $\mathrm{I}, \mathrm{Br}, \mathrm{As}, \mathrm{S}, \mathrm{Se}, \mathrm{Sb}$, and other metalloids. If we take the tube-furnace spectra of King $\ddagger$ to be cases of pure temperature-radiation, we have to admit that at temperatures varying from $2000^{\circ}$ to $3000^{\circ} \mathrm{K}$, most of the alkalies, the alkaline earths, thallium, iron, vanadium, etc., can be made to emit their line-spectra. Gibson § obtained the green line of thallium by simply heating the element contained in a quartz tube. But the conclusion drawn by him-that the intensity of the green line is the same as that of the black body-spectrum at this particular wave-length-is entirely wrong. He placed the quartz tube within a black-body chamber heated to about $1200^{\circ} \mathrm{C}$., and observed that the continuous spectrum from the black body was crossed by a black absorption line corresponding to the green emission line. But this black line faded away as soon as the thallium vapour took up the temperature of the enclosure. From this he concluded that the emission of the green line had just become as intense as that of the black body at the same part of the spectrum. But the conclusion is erroneous, for substances in temperature equilibrium within a black body enclosure would all emit like a black body, and the experiment proves nothing but this simple property of a black-body enclosure.

* Commnnicated by the-Author.

$\dagger$ Pringsheim, Verh. d. Deutsch. Hhys. Gesellschaft, 1903. Wood, Phys. Zeits. viii. (1907), and other papers.

$\ddagger$ King, Astro. Journal, vols. xxviii., xxxiv., $\mathbf{x \times v}$., $\mathbf{x \times x v i i . ~}$

§ Gibson, Phys. Zeits. xii. pp. 1145-1148 (1911). 
According to many physicists, the flame, the arc, and the spark represent gradually increasing stages of temperature, viz. $2000^{\circ} \mathrm{K}, 4000^{\circ} \mathrm{K}$, and $5000^{\circ} \mathrm{K}$. It is well known that spectra produced under these conditions are widely different in their general characteristics, but the hypothesis that these variations can mainly be attributed to the varying values of a single physical variable, viz. the temperature, is not generally accepted.

I wish to point out that the value of the ionization potential, as obtained experimentally by Franck and Hertz, McLennan, and others, or theoretically from the quantum relation $e \mathrm{~V}=h(1, s)$, has a great bearing on the problem. As a rule, the higher the Ionisation-potential of an element, the greater is the difficulty with which it can be excited to emit its linespectrum. 'This will be apparent from the following tables*:

\begin{tabular}{|c|c|c|c|c|c|c|c|c|}
\hline Element.. & $\mathbf{M g}$ & $O_{a}$ & $\mathrm{Sr}$ & $\mathbf{B a}$ & $\mathrm{Na}$ & $\mathbf{K}$ & $\mathbf{R b}$ & $\mathrm{Cs}$ \\
\hline $\begin{array}{l}\text { Ionization } \\
\text { potential. }\end{array}$ & $7 \cdot 65$ & $6 \cdot 12$ & $5 \cdot 70$ & $5 \cdot 21$ & $5 \cdot 11$ & $4 \cdot 32$ & $4 \cdot 16$ & $3 \cdot 88$ \\
\hline Element.. & $\mathrm{Zn}_{\mathrm{n}}$ & $\mathrm{Cd}$ & $\mathrm{Hg}$ & H & $\mathrm{He}$ & $\mathrm{Ne}$ & $A !$ & $\mathbf{N} \ddagger$ \\
\hline $\begin{array}{l}\text { Ionization } \\
\text { potential. }\end{array}$ & $9 \cdot 4$ & 9 & $10 \cdot 45$ & $13 \cdot 6-17 \cdot 1$ & $20 \cdot 5-256$ & $17-23+$ & 16 & $17-18$ \\
\hline
\end{tabular}

The line or lines $\nu=(1, s)-(2, p)$ form the most important lines of an element, and experiments on the ionization potential have shown that when the vapour of an element is bombarded by electrons, this is the line which is the first to be excited, other lines appearing only when the stimulus is substantially greater. The potential $\mathrm{V}=\frac{h}{e}[(1 s)-(2 p)]$ is therefore called the resonance potential, and may be taken to be a measure of the stimulus which is required so that an element may be just excited to radiation of its fundamental line. A better name would probably be "RadiationPotential."

We may give a number of interesting examples. It is well known that generally it is very difficult to excite helium, the smallest trace of a foreign gas tending to quench the He-lines. According to the present theory, this is due to the fact that helium has the highest ionization and radiation potential of all elements, so that when it is subjected to a

* McLennan, Proc. Lond. Phys. Soc.-Guthrie Lecture, Dec. 1918. Franck and IIertz, Verh. d. Deutsch. Ges. vol. xx. (1919).

t Rentschler, Phys. Rev. vol. xiv. p. 503 (1919). Horton and Davies, Proc. Roy. Suc. I.ond., vol. xcriii. p. 124.

$\mp$ Davis \& Goucher, loc. cit. Jan. 1919; also Sunyth, loc. cit. (1919). 
stimulus, this, by preference, passes through the more easily excitable impurities, leaving the $\mathrm{He}$ atoms unaffected. Franck (Zeitschrift für Physik, 1920, vol.i.) describes an interesting experiment on the excitation, by his well-known electronbombardment method, of helium-lines contained in a tube into which a trace of mercury (less than 1 in 1000 parts) was purposely introduced. Mercury has an I.P. of 10.45 volts and R.P. of 4.9 volts, while the corresponding numbers for helium are 25.6 and 20.5 . "We have then," in Franck's own words, "a strong flashing out of the lines of the plement with the lower I.P., at the cost of the lines with the larger I.P." This also explains qualitatively why, with the range of temperatures available in the laboratory, it is not possible by purely thermal means to excite the permanent gases. In the case of the alkalies and the alkaline earths, the value of the ionization potential is low and gradually decreases as we proceed to elements with higher atomic weight in the same group, and it is found that the difficulty with which the spectrum can be excited lessens in a parallel manner. Thus under all conditions, if we classify the alkaline earths according to the ease with which they can be excited, the order is $\mathrm{Ba}, \mathrm{Sr}, \mathrm{Ca}$, and $\mathrm{Mg}$. The same can be said of the other elements.

In the present paper I have used the word "Stimulus" to denote, in a general manner, all physical agencies tending to maks the atoms luminescent. We shall discuss how a high temperature alone can bring about this state. The question can best be approached from the theoretical side.

\section{$\S 2$.}

Modern spectroscopic works have shown that the lines of an element may be grouped under the following headings*:-

(1) Lines due to the normal atom. - In this case the lines are produced by the quantum-changes of orbit of the ontermost electron, the nucleus and the remaining electrons behaving as a single charge. The characteristic Rydberg number in the series-formula is $\mathrm{N}\left(=\frac{2 \pi^{2} e^{4} m}{h^{3}}\right)$. These lines are produced under a comparatively low stimulus.

(2) Lines due to the atom which has lost one electron (the outermost one).- In this case the lines are produced by the quantum changes of orbit of the now outermost electron, the nucleus and the remaining charges behaving as a net

* For example, compare Fowler's work on the "Emission Spectrum of Magnesium," Phil. 'Ĺrans, vol. 214. 
double charge. The characteristic Rydberg number is $4 \mathrm{~N}\left(=\frac{2 \pi^{2} e^{2} m}{h^{3}}(2 e)^{2}\right)$. These lines are produced at a higher stimulus, and generally, but not always ${ }^{*}$, lie in the ultraviolet.

(3) The atom may lose two electrons, and now a new series of vibrations may begin with the new outermost electron. The Rydberg number is $9 \mathrm{~N}$.

The lines of any of these groups are as distinct from each other as if they belong to different elements altogether. According to Sommerfeld $\dagger$, when an atom loses one electron, its spectral properties become similar to those of the element just preceding it in the Periodic Tables. Thus the system of lines of $\mathrm{Ca}^{+}$are constituted in the same manner as those of $\mathrm{K}$; similarly, the relation between $\mathrm{Sr}^{+}$and $\mathrm{Rb}, \mathrm{Ba}^{+}$ and Cs.

Lines of these different groups may be simultaneously present, but generally one group gains in intensity at the expense of the other. Thus in the flame $\mathrm{Ca}^{+}$lines (the $\mathrm{H}$, $\mathrm{K})$ are very faint, the (a-line ( $g$-line) is very strong. Now " $g$ " begins to lose relatively in intensity as the $(\mathrm{H}, \mathrm{K})$ are strengthened with rise of temperature. At the sparkconditions, the most intense lines are the $\mathrm{H}, \mathrm{K}$, while the " $g$ " is almost evanescent.

Let us now examine how the transition from the neutral state to the ionized states (from $\mathrm{Ca}$ to $\mathrm{Ca}^{+}$) takes place, under the influence of heat alone. In Phil. Mag. Oct. 1920 ("Ionization in the Solar Chromosphere," called hencelorth paper A), it has been shown that the problem can be attacked with the aid of the "New Thermodynamies" of Planck and Nernst $\ddagger$, and the statistical equilibrium between neutral atoms and ionized atoms can be calculated in terms of temperature and pressure, when the energy of innization is known. The calculations for alkaline earths will be found in paper $A$, and those for the alkalies are given in paper $B$ \&.

According to the mechanical theory, the outermost electron of the neutral atom revolves in a stable orbit when the atom is not subject to any stimulus. Ionization means the transference of this electron to infinity. But the process

* For example, in case of alkaline earths. All alkaline earths are distinguished by having large $(2, p)$ terms, which causes the principal lines to occur in the visible region.

† Sommerfeld, Atombau und Spektralanalyse, Chap. 4, \$6 and Appendix.

I Planck, Wärmestrahlung. Nernst, Das Neue Wärmesatz, etc.

\$ "Elements in the Sun," Phil. Mag. Dec. 1920. 
is not an abrupt one, for, according to the quantum theory of spectral emission, the electron may have an infinite number of orbits distinguished by different quanta-numbers.

The theory of the stable orbits has been formulated by Sommerfeld ${ }^{*}$ in the following manner. Let $n_{1}, n_{2}$ denote the rotational quantum numbers for an orbit, and $n^{\prime}$ the radial quantum number, i. e. $r, \theta, \phi$ being the coordinates of the electron; then,

$$
\int\left(\frac{\partial^{\prime} \mathrm{T}}{\partial r}\right) d r=n^{\prime} h, \quad \int\left(\frac{\partial \mathrm{T}}{\partial \dot{\theta}}\right) d \theta=n_{1} h, \quad \int\left(\frac{\partial \mathrm{T}}{\partial \dot{\phi}}\right) d \phi=n_{2} h,
$$

the integration extending over the whole orbit. The energy of the system is now given by the expression

$$
\mathrm{A}-h\left[\frac{\mathrm{N}}{\left\{n+n^{\prime}+f\left(n_{1}, n_{2}, n^{\prime}\right)\right\}^{2}}\right], \quad n_{\mathrm{I}}+n_{2}=n,
$$

where $\mathrm{A}=\mathrm{a}$ constant, $f\left(n, n^{\prime}\right)$ is a function, the value of which decreases with increasing values of $n$ and $n^{\prime}$.

The possible orbits can now be thus classified by assigning different sets of values to $n$ and $n^{\prime}$.

TABLe II.

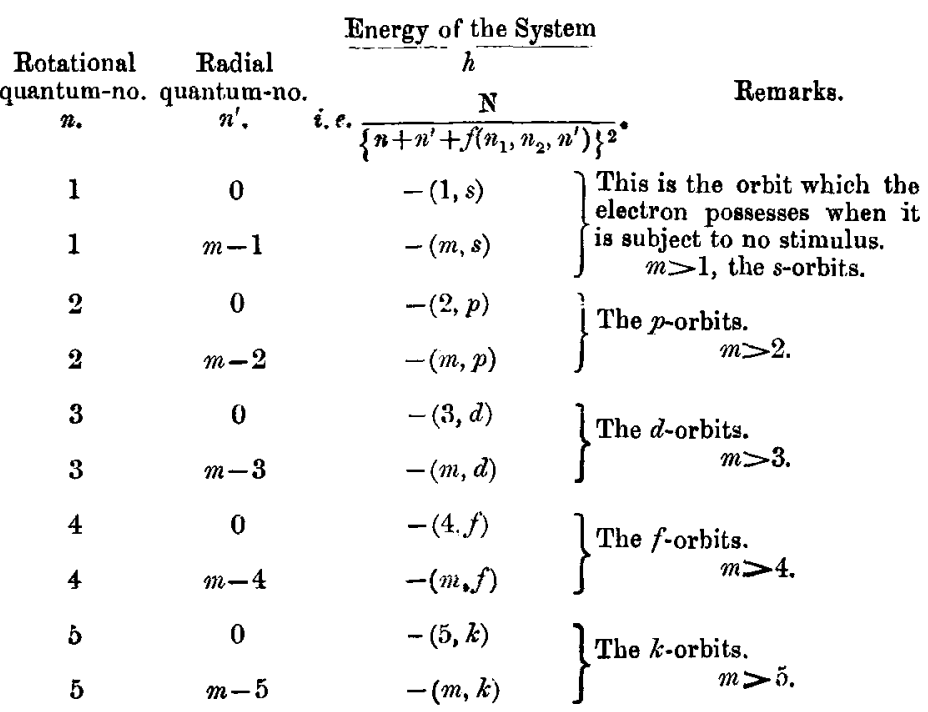

$(1, s),(2, p),(3, d), \ldots(m, s), \ldots$ are the familiar expressions which, in Paschen's notation, denote the terms of which a

* Sommerfeld, Verh. d. Deutsch. Phys. Ges, vol, xxi. (1919). 
series-formula is composed. Thus, for the principal series, the series-formula is

$$
\nu=(1, s)-(m, p), \quad m \geqslant 2 .
$$

When the atom is in a free gaseous condition, and is not subject to any stimulus, it has the energy $\mathrm{A}-h(1, s)$. The ligher orbits are produced only when a stimulus is applied. The lines are emitted as the electron changes its habitat from one stable orbit to another with less energy. Thus the line $(1, s)-(2, p)$ is emitted when the electron changes its habitat from the $p$-orbit $(2, p)$ to the $s$-orbit $(1, s)$, otc. The law according to which these changes take place has been thus formulated by Rubinowicz * and Bohr. Let $n_{0}$ and $n$ denote the rotational quantum numbers of the initial and final orbits. Then

$$
n-n_{0}=1,0 \text {, or }-1 \text {. }
$$

We can thus distinguish among the following different types of combination:-

Group I.-Positive combination, $n-n_{0}=1$.

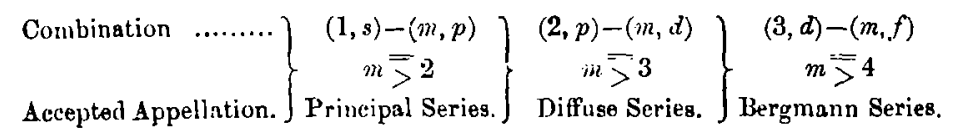

$$
\begin{array}{ccc}
(2, s)-(m, p) & (3, p)-(m, d) \\
m>3 . & m>4 . & \text { etc. }
\end{array}
$$

Group II.-Negative combination, $n-n_{0}=-1$.

Combination

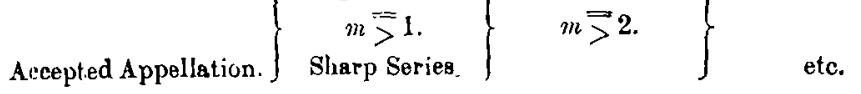

Grovp III.-Neutral combination.

Oombination $\ldots \ldots \ldots . . . \quad(1, s)-(m, s) \quad(2, p)-(m, p) \quad(3, d)-(m, d)$

Each of the terms $(m, s),(m, p),(m, d)$ may have double or multiple values owing to the different possible orientations of the vibrating electron with regard to the remaining atom $\dagger$.

- Rubinowicz, Phys. Zeits. vol. xix.pp. 44l-4e5 (1918). Sommerfeld, Atombau und Spektralanalyse, pp. 390-403.

+ For a theoretical treatment of the case, see S. N. Basu, "On the Deduction of Rydberg's Law from the Quantum Theory of Spectral Enission," Phil. Mag. Nov. 1919. 
Thus far we have dealt with the electrodynamics of the atomic system, i.e. the possible stable orbits of the vibrating electron calculated according to quantum-mechanics. We have now to deal with the statistics of the case, for the higher stable orbits are produced in sufficient proportion only with increasing stimulus, which we obtain only at higher temperatures. The intensity of a line will depend upon the product of the numbers which show the relative proportions of orbits in the initial and final stages at any instant, and also upan the chance of changing from one orbit to another. Thus the intensity of $(1, s)-(2, p)$ will be proportional to $\lambda \lambda^{\prime} \epsilon$, where $\lambda$ is the number of orbits in state $(1, s), \lambda^{\prime}$ is the number in state $(2, p)$, and $\epsilon^{\prime}$ is the chance that the orbit will change from the state $(2, p)$ to $(1, s)$.

When the stimulus is sufficiently great, some of the electrons will pass off to infinity, and we shall have partial ionization. This problem can be treated thermodynamically, for here we have to consider a sort of chemical equilibrium between three distinct phases- the neutral atom, the ionized atom, and the electron. But radiation cannot be so treated, for this is a case of internal change of orbits only, not involving any phase-changes.

The problem before us can therefore be thus stated:- How to find out the statistical distribution of atoms into different possible stable orbits when the mass of the gas is subjected to a given thermal stimulus?" According to the theory of ionization sketched in paper $\mathrm{A}$, if Ca-vapour is enclosed in a vessel, such that the pressure is always maintained at $10^{-3} \mathrm{~atm}$., we have seen that with increasing temperature the proportion ionized varies as follows :-

$\left.\begin{array}{ccccccc}\text { Temperature } \ldots & 2000^{\circ} & 3000^{\circ} & 4000^{\circ} & 5000^{\circ} & 6000^{\circ} & 7000^{\circ} \\ \begin{array}{c}\text { Percentage of } \\ \text { Ionization } \ldots\end{array} & 3 \times 10^{-4} & 2 \times 10^{-1} & 6 & 47 & 91 & 100\end{array}\right\}$

The non-ionized atoms cannot all be with the primitive orbit $(1, s)$, but a good proportion will be found with the other possible stable orbits, for the electron, while detaching itself from the neutral atom, lias a chance of taking its habitat in some of these stable orbits, and hence some must be found in these states. The phenomena of lineradiation therefore comes before the ionization becomes complete.

Phil. Mag. S. 6. Vol. 41. No. 242. Feb. 1921. 
$\$ 3$.

At the present time, the electrodynamical part, -i. e., the manner in which the lines of an element originate from the quantum-vibrations of the constituent electrons of the atomic system-has been worked up in the case of the $\mathrm{H}$-atom alone. For the other atoms, it is only in the process of making*. But the thermodynamical part of the problemthat is, how the proportion of different possible stable orbits varies according to temperature, and how the orbits change from one into the other giving rise to line-radiationshas not even been clearly formulated. In his interesting development of the quantum theory applied to systems with more than one degree of freedom, Planckt has laid the foundations of a new method for dealing with the statistical aspect of the question. The second aspect-namely radiation following as a result of mutual interchange of stable orbitshas been dealt with by Einstein $\ddagger$. But there are still many difficulties to overcome. When this is done, a now and fruitful chapter-that of line radiation of gases-will be added to thermodynamies.

We may provisionally proceed along the following lines:Let us suppose that the orbits having the rotational quanta $1,2, \ldots m \ldots$ vary as a geometric progression

$$
(1-f)\left[1, f, f^{2}, f^{3} \ldots\right]
$$

where $f$ is a fraction and a function of temperature and concentration. Similarly, let us suppose that the orbits having the radial quanta $0,1,2,3$, etc., vary according to the terms of the geometric progression

$$
(1-g)\left[1, g, g^{2}, g^{3} \ldots\right],
$$

where $g$ is a similar fraction.

Then at any instant the proportion of orbits with the rotational quantum number $n+1$ and the radial quantum number $n^{\prime}$ is

$$
f^{n} g^{n^{\prime}}(1-f)(1-g)
$$

since at low temperatures and high concentrations almost the whole number of atoms is in the state $(1, s)$ corresponding to $n=1, n^{\prime}=0$.

We see that $f$ and $g$ are very small quantities under these

* Sommerfeld, Atombau und Spektralanalyse.

+ Planck, Verh. d. D. Phys. Ges. vol. xrii. p. 407 (1915); Ann.d. Phys. vol. 50. p. 385 (1916).

† Einstein, Verh. d. D. Phys. Ges. vol. x.viii. p.318 (1916); Phys. Zeit. 1918, p. 124. 
conditions and gradually increase as the temperature is raised or the concentration is lowered. As an example, we may take that the distribution of the Ca atoms in the orbits at $2000^{\circ} \mathrm{K}$ follow according to the scheme (we are considering only $f$ here)

$$
\left(1-\frac{1}{10}\right)\left[1,10^{-1}, 10^{-2}, 10^{-3} \ldots\right]
$$

while at $4000^{\circ} \mathrm{K}$, the distribution is

$$
\left(1-\frac{1}{2}\right)\left[1, \frac{1}{2},\left(\frac{1}{2}\right)^{2},\left(\frac{1}{2}\right)^{3} \ldots\right] \text {, }
$$

so that the relative intensity of the lines

$$
\begin{aligned}
& \text { will be }(1, s)-(2, p), \quad(2, p)-(3, d), \quad(3, d)-(4, b) \\
& 1: 10^{-1}: 10^{-2}, \text { etc.; }
\end{aligned}
$$

but fully 80 per cent. of the atoms will remain inactive, while at $4000^{\circ} \mathrm{K}$, the ratio will be

$$
1: \frac{1}{2}:\left(\frac{1}{2}\right)^{2} \text {, }
$$

but now only 25 per cent. of the non-ionized atoms remain inactive.

The above considerations are not hased upon any theoretical argument, but are given here as a sort of construibar vorstellung of the statistics of line-radiation of gases. We can say that, under all physical conditions, a very small concentration of radiant atoms suffices for the production of the series $\nu=(1, s)-(m, p)$, especially the fundamental line $\nu=(1, s)-(2, p)$. The $(2, p)-\left(m, d_{i}\right),(2, s)-(m, p),(3, d)$ $-(n, \tau)$ lines require gradually increasing concentrations of radiant matter in addition to the condition that the stimulus should be sufficiently great. For example, if we take sodium gas at a temperature of $1500^{\circ} \mathrm{K}$ [an ordinary Bunsen flame tinged with sodium vapour], and gradually decrease the amount of vapour in the flame, the order in which the lines disappear are $(3, d)-(m, b),(2, s)-(m, p), \quad(2, p)$ $-(m, d) ;$ the $(1, s)-(m, p)$ lines, of which the leading members are the $D_{1}$ and the $D_{2}$, are the last to disappear, a fact which was recognized by Du Gramont, when he conferred the appellation "raies ultimes" upon this class of lines*. This state of affairs persists when the temperature is raised and gradually increasing percentages of atoms are ionized. If a line is represented by the series formula $\nu=(n, f)-\left(n^{\prime}, f^{\prime}\right)$,

* According to Bunsen and Kirchhoff, $7 \times 10^{-12} \mathrm{gm}$. of sodium in the Bunsen flame is quite sufficient to show the $\mathrm{D}_{1}$ and the $\mathrm{D}_{2}$ line (Pringsheim, Physik der Sonne, p. 121). 
the difficulty of detection of the line will be greater the larger are the values of $n$ and $n^{\prime}$.

In the following table we give the temperature of complete ionization of a few elements, with the temperatures at which luminescence just begins and attains its maximum intensity. But it will be clear from what has been said that the ordinary way of speaking_-" the gas is heated to incandescence or luminescence just begins" - has no meaning in itself unless we say which particular line is emitted, or which orbit is produced. The orbits which are produced are specified in column 3. Under the heading "Remarks," the manner in which these temperatures have been estimated are briefly described. In this connexion, the following section on absorption should be consulted. The pressure has been taken equal to one atmosphere unless otherwise stated.

TABLE III.

\begin{tabular}{|c|c|c|c|c|c|c|}
\hline Element. & $\left\{\begin{array}{c}\text { Temperature } \\
\text { of } \\
\text { Ionization. }\end{array}\right.$ & $\begin{array}{c}\text { Orbit } \\
\text { generated. }\end{array}$ & $\begin{array}{l}\text { Lumin- } \\
\text { escence } \\
\text { begins at }\end{array}$ & Remarks. & $\left\{\begin{array}{c}\text { Luwin- } \\
\text { escence is } \\
\text { maximum } \\
\text { at }\end{array}\right.$ & Remarks. \\
\hline $\mathrm{H} \quad \ldots .$. & 24,000 & $(2, p)$ & $5000^{\circ} \mathrm{K}$ & $\begin{array}{l}\text { Temp. of } \\
\text { Ma stars }\end{array}$ & $12,000^{\circ} \mathrm{K}$ & $\begin{array}{l}\text { Temp. of } \\
\text { Aostars }\end{array}$ \\
\hline $\mathrm{He} \ldots$ & 32,000 & $(2, p)$ & $11,000^{\circ} \mathrm{K}$ & $\begin{array}{l}\text { Temp. of } \\
\text { A2 class }\end{array}$ & $17,000^{\circ} \mathrm{K}$ & $\begin{array}{l}\text { Temp. of } \\
\text { B2A class }\end{array}$ \\
\hline $\mathrm{Mg}^{+}$ & 24,000 & $(3, d)$ & $7500^{\circ} \mathrm{K}$ & $\begin{array}{l}\text { Temp. of } \\
\text { Go class }\end{array}$ & $11,000^{\circ} \mathbf{K}$ & $\begin{array}{l}\text { Temp. of } \\
\text { A2F class }\end{array}$ \\
\hline $\left.\begin{array}{l}\mathrm{Ca} \\
\mathrm{Sr} \\
\mathrm{Ba}\end{array}\right\}$ & $\begin{array}{c}10,000- \\
8000^{\circ} \mathrm{K} \\
(\text { Pressure } \\
\left.10^{-1} \text { atm. }\right)\end{array}$ & $(2, p)$ & $1500^{\circ} \mathrm{K}$ & $\begin{array}{c}\text { Bunsen } \\
\text { flame }\end{array}$ & 4000 & $\begin{array}{c}\text { The open } \\
\text { arc }\end{array}$ \\
\hline
\end{tabular}

The elements $\mathrm{N}_{2}, \mathrm{O}_{2}, \mathrm{~A}, \mathrm{Ne}$, etc., resemble $\mathrm{H}_{2}$ and $\mathrm{He}$ in having large values for the ionization-potential, and therefore they fail to respond to the temperatures which can be commanded in the laboratory. The alkali metals (particularly $\mathrm{K}, \mathrm{Rb}, \mathrm{Cs}$ ) are more prominent in the flame-spectra and less in the arc than the alkaline earths. Mg-lines are rather faint in the flame, but come out very prominently in the open arc. $\mathrm{Zn}, \mathrm{Cd}, \mathrm{Hg}, \mathrm{Fe}$, Ti lie between the alkaline earths and the permanent gases in their spectral properties. All this is in very good qualitative agreement with the hypothesis sketched in the present paper. 


\section{$\S 4$. Absorption.}

In this connexion, we may consider the puzaling question of reversal of lines. According to Kirchhoft's law, we expect that the emission-lines of an element should be reversed when a strong beam of white light is sent through cooler lavers of the vapour. But this expectation is not always fulfilled. Wood* has found that if a white light be sent through a column of sodium vapour, only the lines of the principal pair-series $(1, s)-\left(m, p_{1}\right),(1, s)-\left(m, p_{2}\right)$, can be obtained as absorption-lines. None of the lines of the diffuse or the sharp series are reversed. Bevan $\dagger$ has extended the method to the other alkali metals, i. e. Potassium, Rubidium, and Cæsium, and arrived at identical results. Recently Dobbie and Fox (Proc. Roy. Soc. vol. xeviii. p. 147) studied the ahsorption-spectra of $\mathrm{Hg}, \mathrm{Zn}$, and $\mathrm{Cd}$ vapour, and found no absorption up to $\lambda=3200$. But this is due to the fact that the $(1, s)-(m, p)$ lines of these elements lie below $3000 \AA . U$. In fact, Wood found in 1913 (Phys. Zeit. pp. 191-195) that ordinary $\mathrm{Hg}$ vapour absorbs the fundamental line $\lambda=2536$ $(1, s)-\left(2, p_{2}\right)$.

The explanation easily follows from our theory. The condition for absorption is that in the atoms present, there should be a fairly large number with orbits corresponding to the first term of the pulse of radiation to be absorbed. Thus, in order that a line $(2, p)-(m, d)$ may be absorbed, we must have a sufficient number of atoms with $(2, p)$ orbits. At low temperatures only atoms with $(1, s)$ orbits are present. Hence only the lines corresponding to the combination $(1, s)-(m, p)$ are absorbed. The lines represented by the positive combinations $(2, p)-(m, d)$, $(3, d)-(m, \dot{f})$, or the negative combinations $(2, p)-(m, s)$, can only occur when atoms with $(2, p)$ or $(3, d)$ orbits are present. This can happen only at high temperature or under electrical stimulus.

The temperature required for this purpose is very highmuch higher than the temperatures used by Wood and Bevan for all elements. In fact, the atoms begin to absorb the lines $(2, p)-(m, d)$ only when they are hot enough to emit the leading terms of the principal series. A line of the Bergmann series will begin to be absorbed at even a higher temperature, viz. at the temperature of emission of the diffuse series $(3, d)-(4, b)$.

If the views presented here be correct, we may probably obtain the reversal of the lines of the diffuse or the Bergmann

* Wood, The Astrophys. Journal, vol. xxix. pp. 97-100.

+ Bevan, Proc. Roy. Soc. vol. lxxxiii. pp. 423-428; vol. lxxxv. pp 58-76. 
series of the alkali metals by heating the absorbing column of vapour to about $2000^{\circ}$ to $3000^{\circ} \mathrm{K}$. The most favourable element to start with is cesium, which has the lowest ionization-potential of all elements.

In many cases confusion may arise about the proper identification of the $(1, s)-(m, p)$ terms. Thus, what are usually called the Principal series of helium and parhelium (viz. the series beginning with the line 20,587 for parhelium and 10,834 for helium) do not really correspond to the combination $(1, s)-(m, p)$, but to the combination $(2, s)$ $-(m, p)$. The $(1, s)$ term for helium is still unknown, and the series $(1, s)-(m, p)$ lie far down in the ultraviolet*. Hence, according to our theory, none of the lines belonging to the combinations $(2, s)-(m, p),(2, p)-(m, d)$ can be absorbed by a layer of heliam gas.

But if by heating or some other means we can convert a good proportion to the states $(2, s)$ or $(2, p)$, then and then only can these lines appear as absorption-lines. But at a pressure of $10^{-1} \mathrm{~atm}$. helium becomes incandescent, $i$. $e$. emits the lines $(1, s)-(m, p)$, and absorbs the lines $(2, p)$ $-(m, d)$ at probably not less than $11,000^{\circ}$ or $12,000^{\circ} \mathrm{K}$, i. $e$. only in stars of the B-class.

But instead of a high temperature we may think of other means. The spark produces mechanically the very same conditions which can be realized at very high temperatures. This is exactly what Paschen $\dagger$ has done. He found that the lines of the combination $(2, s)-(m, p)$ for helium and parhelium cannot be absorbed by an ordinary layer of the helium gas. But when a spark is sent through the absorbing layer the lines are strongly absorbed, the absorbed energy being again re-emitted in all directions.

The paper thus suggests more problems than it attempts to solve. A critical examination and further development of the hypothesis advanced here requires an overhauling of the whole data on the line-radiation of gases-such as are contained in Kayser's Handbuch der Spektroskopie and further works. But this programme requires much more time and more extensive study, both practical and theoretical.

University College of Science, Calcutta, May 25, 1920.

* It is quite possible that some of the $(1, s)-(m, p)$ lines for helium and parhelium may be identical with the lines discovered by Lyman in the ultra-violet, and some with the lines discovered by Richardson and Bazzoni in the region of 300 to $400 \mathrm{~A}$.U. by the photo-electric method (vide Richardson and Bazzoni, Phil. Mag. 1918).

$\dagger$ Paschen, Ann. d. Physik, vol. xlv. p. 625 (1914). 